\title{
Preshaped Visual Grasp of Unknown Objects with a Multi-Fingered Hand
}

\author{
Vincenzo Lippiello, Fabio Ruggiero, Bruno Siciliano and Luigi Villani
}

\begin{abstract}
In this paper a method for fast visual grasp of unknown objects with a multi-fingered hand is presented. The algorithm is composed of an object surface reconstruction algorithm and a local grasp planner, evolving in parallel. The former uses an elastic reconstruction surface, whose dimensions are assigned initially by a preshaping process, and which shrinks toward the object until some parts of the surface intercept the object visual hull. The latter moves the fingertips on the current available reconstruction surface towards points which are optimal (in a local sense) with respect to a certain number of indices weighting both the grasp quality and the kinematic configuration of the hand. Experiments are presented, showing the effectiveness of the proposed algorithm.
\end{abstract}

\section{INTRODUCTION}

Grasping and manipulation tasks typically require a priori knowledge about the environment and the objects geometry. The adoption of vision can be useful to reduce the need of such information. Nowadays, autonomous grasping operation in unstructured environments is a challenging research field.

Generally, two main tasks have to be performed to achieve unknown objects grasping: object reconstruction and grasp planning. But also the preshaping of a robotic hand -the preparation of the hand to grasp the object- is a nontrivial step to grasping, as described in [14]. In the literature, several methods deal with the preshaping problem, most of them relying on a previous knowledge learned from humans [20]. Others rely on the use of vision [21], adopting a variety of approaches such as fuzzy logic [1] or box-based approximation [10], and generally are task-dependent [17].

One of the first approaches to the grasping problem in unknown environments can be found in [22]. Afterwards, different methods have been proposed in the literature to cope with 3D model reconstruction of unknown objects. The main differences depend on how the available object images are processed and on the algorithms used for object reconstruction. A certain number of algorithms can be classified under the so called volumetric scene reconstruction approach, while others are referred as surface scene reconstruction algorithms [8]. A method to grasp an unknown object using information provided by a deformable contour model

The research leading to these results has been supported by the DEXMART Large-scale integrating project, which has received funding from the European Community's Seventh Framework Programme (FP7/2007-2013) under grant agreement ICT-216239. The authors are solely responsible for its content. It does not represent the opinion of the European Community and the Community is not responsible for any use that might be made of the information contained therein.

The authors are with PRISMA Lab, Dipartimento di Informatica e Sistemistica, Università degli Studi di Napoli Federico II, via Claudio 21, 80125, Naples, Italy \{lippiello, fabio.ruggiero, siciliano, lvillani\}@unina.it algorithm is proposed in [15]. A finite-elements approach is used in [5] to reconstruct both $2 \mathrm{D}$ and $3 \mathrm{D}$ object boundaries where, using an active contour model, the data extracted from images are employed to generate a pressure force on the contour that inflates or deflates the curve, like a balloon.

On the other hand, grasp planning techniques rely upon the choice of grasp quality measures used to select suitable grasp points. Several quality measures proposed in the literature depend on the position of the contact points (algebraic properties of the grasp matrix, geometry of the grasp area of the polygon created by the contact points and so on), while other depend on the finger forces. A rich survey of grasp quality measures can be found in [19]. In [9] two optimal criteria are introduced, where the total and the maximum finger force are considered, while in [11] measures based on algebraic properties of the grasp matrix and a measure based on the task to accomplish are presented. Moreover, measures depending on the hand configurations [18] define a set of quality measures based on the evaluation of the capability of the hand to realize the optimal grasp. However, planning grasp configurations for a robotic hand should take into account quality measures depending both on grasp geometry and on hand configuration as addressed in [2], [4].

A method for fast visual grasping of unknown objects using a camera mounted on a robot in an eye-in-hand configuration is here presented. This method is composed of an object surface reconstruction algorithm and of a local grasp planner, which evolve in a parallel way. Differently from the method described in [12], a new preshaping stage has been introduced, the reconstruction algorithm has been revisited and the local grasp planner has been completely changed, being now suitable for a multi-fingered grasp.

\section{FLOATING VISUAL GRASP ALGORITHM}

The classical serial approach in grasping objects of unknown shape consists of two stages: in the first the object is completely reconstructed, while the evaluation of the optimal grasp under a selected global criterion starts at the end of this stage, as showed in Fig. 1. This approach gives the best results in terms of the grasp quality since the evaluation is made in a global way. However, the total execution time is given by the sum of the time due to the object reconstruction and the time due to the grasp synthesis and planning. Although the modern technologies allow a fast object reconstruction, the investigation of all the possible combinations of the grasp points or of the set of surfaces which approximate the object (depending on the reconstruction method adopted) 


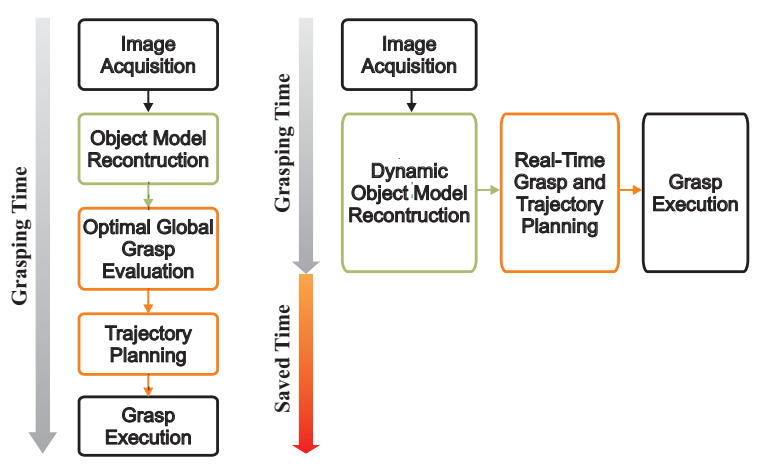

Fig. 1. Classical serial method vs. proposed parallel approach.

could generally require a considerable amount of time. Obviously, this drawback is irrelevant for off-line applications, but it could be unsuitable for real-time grasping, if powerful hardware is unavailable. The proposed parallel approach may represent a valid alternative in such cases, where the total computational time is given by the slower between the reconstruction and the planning stage (see Fig. 1). In fact, these two parallel processes are independent and can be allocated under different computational resources. The main drawback of the proposed parallel approach is that the final grasp is optimal only in a local sense.

The elaboration processes may be arranged into three main groups: image acquisition and preshaping, object surface reconstruction algorithm, and local grasp planner.

During the first stage a set of $n$ images suitable for the reconstruction process is acquired. Then, the silhouette of the object for each acquired image is evaluated, and the object center of mass, assuming a homogeneous mass distribution, is estimated using a least-squares triangulation method [12].

The preshaping algorithm, as explained in the next section, starts to compute, from the bounding box of each silhouette, a polyhedron in the Cartesian space, which represents a polyhedric overestimation of the visual hull. Then, the initial reconstruction surface, with elliptical shape and centered at the estimated center of mass of the object, is built on the basis of the dimension of the polyhedron. Further, the initial grasp configuration of the hand is evaluated, which depends on the initial reconstruction surface.

After this preshaping step, both the object model reconstruction process and the local planner start in parallel and cooperate to the final goal. In particular, the reconstruction algorithm updates in real-time the estimation of the current reconstructed object surface, while the local planner, on the basis of the current estimation, computes the fingertips trajectories toward a local optimal grasp configuration.

The assumptions made throughout the paper are that an eye-in-hand configuration with a calibrated camera is available for the reconstruction stage. The observed object has to be fixed in the space during the images acquisition and distinguishable with respect to the background and other objects - from a topological point of view, the object must be an orientable surface with genus 0 .

\section{PRESHAPING}

The proposed preshaping method starts from a concept proposed in [7], where the evaluation of a rough shape estimation is made by a linear programming technique. For each of the $n$ silhouettes and from the relative bounding box, one can build four planes in the Cartesian space, each containing the camera origin and two adjacent vertices of the corresponding silhouette's bounding box, resulting in $4 n$ Cartesian planes. Obviously, each plane splits the Cartesian space into two regions, one of which contains the visual hull. The intersections of all these planes create a polyhedron $\mathcal{P}$ which contains the object visual hull, or in other words, is a polyhedrical overestimation of this last.

The vertices of this polyhedron can be quickly computed by solving a linear programming problem. Since each side of each bounding box is associated with a plane, if the normal unit vector to the plane is chosen pointing outwards with respect to the interior side of the bounding box, the inner space of this plane is represented by the set of inequalities:

$$
A_{i} \boldsymbol{x} \leq \boldsymbol{d}_{i}
$$

where subscript $i$ denotes the $i$-th image, with $i=1, \ldots, n$, $A_{i}$ is a $4 \times 3$ matrix whose rows are the transpose of the normal unit vectors, and $\boldsymbol{d}_{i}$ is a $4 \times 1$ vector whose elements define uniquely the positions of the planes in the space. Stacking all the $A_{i}$ and $\boldsymbol{d}_{i}$ in the matrices $A$ and $\boldsymbol{d}$, the inner space of the polyhedron is represented by:

$$
A \boldsymbol{x} \leq \boldsymbol{d}
$$

Considering (1) as a set of constraints in a minimization problem, the vertices of the corresponding polyhedron are the so called basic feasible solutions, whose computation is well known in literature. Notice that, since the problem has been formulated as a linear programming problem, the computational time is very short and it depends only on the number $n$ of images.

Once all the $n_{v}$ vertices $\boldsymbol{x}_{v}=\left[\begin{array}{lll}x_{v_{x}} & x_{v_{y}} & x_{v_{z}}\end{array}\right]^{T}$ of the polyhedron $\mathcal{P}$ have been computed, the central moments can be evaluated as:

$$
\mu_{i, j, k}=\sum_{\boldsymbol{x}_{v} \in \mathcal{P}}\left(x_{v_{x}}-\bar{x}_{v_{x}}\right)^{i}\left(x_{v_{y}}-\bar{x}_{v_{y}}\right)^{j}\left(x_{v_{z}}-\bar{x}_{v_{z}}\right)^{k}
$$

where $\overline{\boldsymbol{x}}_{v}=\left[\begin{array}{lll}\bar{x}_{v_{x}} & \bar{x}_{v_{y}} & \bar{x}_{v_{z}}\end{array}\right]^{T}=\frac{1}{n_{v}} \sum_{i=1}^{n_{v}} \boldsymbol{x}_{v_{i}}$.

Finally, a pseudo-inertia tensor of the polyhedron can be defined as:

$$
I=\left[\begin{array}{lll}
\mu_{2,0,0} & \mu_{1,1,0} & \mu_{1,0,1} \\
\mu_{1,1,0} & \mu_{0,2,0} & \mu_{0,1,1} \\
\mu_{1,0,1} & \mu_{0,1,1} & \mu_{0,0,2}
\end{array}\right]
$$

where its eigenvalues and eigenvectors define the principal axes of inertia of an ellipsoid, which is employed here as the initial shape of the reconstruction surface. Finally, the ellipsoid is suitably enlarged ensuring that the object is wrapped, as shown in the examples of Fig. 2.

Depending on the object shape, the ellipsoid may have one axis bigger than others, one axis smaller than others, or 


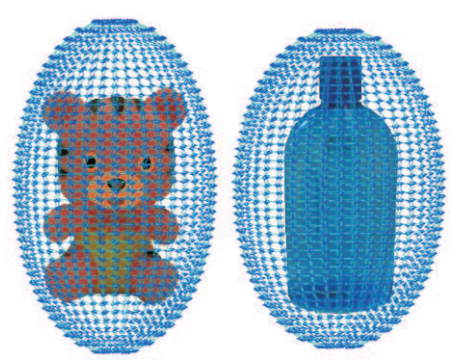

Fig. 2. Examples of preshaped elliptical reconstruction surfaces.
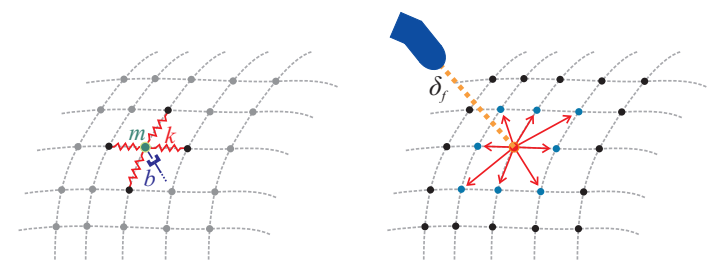

Fig. 3. Cross network topology of the reconstruction surface (on the left), and the contour of neighbor points (on the right).

all axes of a similar dimension. For all these cases, a good choice for the grasp configuration depends also on the task to accomplish (e.g. pick-and-place, manipulation, assembling, etc.), on the type of grasp to perform (firm or fine), on the environmental constraints (e.g. the ground plane), and on the hand kinematics and the number of fingers. When firm grasp is considered, the axis of approach of the hand is typically chosen parallel to the biggest axis of the ellipsoid. On the other hand, for the fine manipulation case, the axis of approach depends from several factors. In this paper, for simplicity and considering the previous assumptions of fine manipulation, the initial grasp configuration is chosen as an equilateral grasp in a plane parallel to the two minor axes of the ellipsoid, when it is reachable with respect to the hand and environmental constraints.

\section{OBJECT SURFACE RECONSTRUCTION}

As described in the previous sections, from the set of $n$ silhouettes of the object, an elliptical initial reconstruction surface is generated, virtually placed around the object and sampled by $n_{s}$ reconstruction points. A virtual mass is associated to each point, and four links are imposed by springs connecting the closest cross points, resulting in a cross reticular topology (see Fig. 3).

Each parallel of the ellipsoid should have the same number $n_{m}$ of points, corresponding to the number of meridian, allowing the construction of a fully linked cross reticulum. In other words, for each point, the existence of a couple of corresponding points on the closest parallels of the grid is guaranteed. Without loss of generality, the number $n_{p}$ of parallels is chosen equal to the number of meridians $n_{p}=n_{m}=\sqrt{n_{s}-2}$.

The model of the system, defining the deformation motion of the reconstruction surface, is described by the following

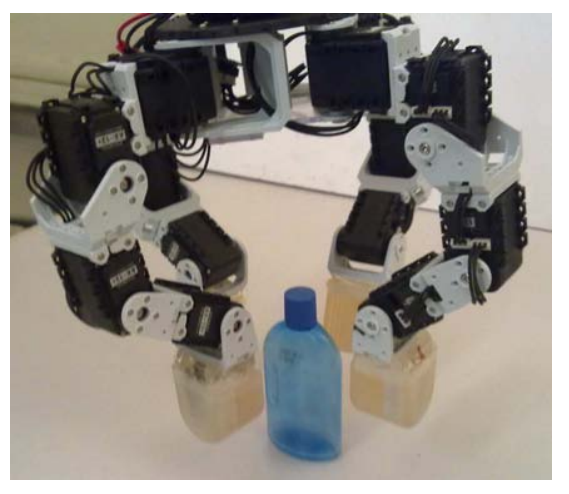

Fig. 4. Floating visual grasp.

dynamic equations:

$$
m \ddot{\boldsymbol{x}}_{i, j}+b \dot{\boldsymbol{x}}_{i, j}+k\left(4 \boldsymbol{x}_{i, j}-\boldsymbol{c}\left(\boldsymbol{x}_{i, j}\right)\right)=\boldsymbol{f}_{i, j},
$$

for $i=1, \ldots, n_{m}$ and $j=1, \ldots, n_{p}$, where $\boldsymbol{c}\left(\boldsymbol{x}_{i, j}\right)=$ $\boldsymbol{x}_{i-1, j}+\boldsymbol{x}_{i, j+1}+\boldsymbol{x}_{i+1, j}+\boldsymbol{x}_{i, j-1}$, and $\boldsymbol{x}_{i, j}$ is the position in the workspace of the sampling point at the intersection of the $i$-th meridian with the $j$-th parallel. The parameters $m, k$, and $b$ represent the mass associated to the point, the constant spring linking the point with its nearest four cross points, and the constant spatial damper, respectively.

Vector $\boldsymbol{f}_{i, j}$ is the reconstruction force acting on the mass associated to the sample point $(i, j)$, which is attractive with respect to the border of the visual hull and is progressively reduced every time the corresponding point comes in or goes out from the visual hull:

$$
\boldsymbol{f}_{i, j}=\alpha_{i, j}\left(t_{i, j}\right) F_{a} \boldsymbol{n}_{i, j}
$$

where $\boldsymbol{n}_{i, j}$ is the unit vector pointing from the current point $(i, j)$ to the estimated centroid of the object, and the term $\alpha_{i, j}\left(t_{i, j}\right) F_{a}$ is the amplitude of the force. In detail, $F_{a}$ is the maximum amplitude of the force and $\alpha_{i, j}\left(t_{i, j}\right) \in(-1,1]$ is a discrete sequence of scale factors defined as follow:

$$
\alpha_{i, j}\left(t_{i, j}\right)=-\epsilon \alpha_{i, j}\left(t_{i, j}-1\right),
$$

where $\epsilon \in(0,1), \alpha_{i, j}(0)=1$, and $t_{i, j}$ is an integer index which starts form zero and is incremented every time the point $(i, j)$ comes in or goes out the visual hull.

\section{LOCAL GRASP PLANNER}

The current estimation of the object surface is stored in a proper buffer, which is continuously updated during the dynamic evolution of the elastic surface, and is employed by the local grasp planner for updating the fingertips trajectories. The local grasp planner, in accordance with the current reconstructed object surface, generates the fingertips trajectories on the basis of suitable quality indices, but keeping a fixed floating safety distance $\delta_{f}$ between the fingertips and the corresponding reconstruction points, along the outgoing normal to the surface (see Fig. 4). The distance is exploited like a security parameter to avoid undesired collision between the fingers and the object before the final grasp. 
Namely, starting from the initial grasp configuration, the planner generates the motion of the fingertips from the current position to a new set of points of the updated surface, according to a force field associated at each contact point, until no improvements in the quality of grasp are reached. This new configuration of the contact points will be the new initial grasp configuration for the next iteration of the local grasp planner. The process ends when the object reconstruction algorithm reaches an equilibrium and the planner computes the final grasp configuration.

The quality indices employed to generate the force field and the finger trajectory planner are presented in the follows.

\section{A. Motion field of forces}

In this paper planar grasps in the 3D space are considered, assuming that the moments and transversal forces acting on the object can be neglected. In particular, the desired optimal grasp is characterized by having all the contact points lying on the same grasping plane in an equilateral configuration [13], [19]. This choice guarantees force closure for a large number of situations and simplifies the computation of good grasps, although it may exclude a number of grasp configurations that can be more effective. Moreover, the area of the grasp polygon, resulting from the projection of the contact points on the grasp plane, has to be maximized to improve the quality of the grasp with respect to possible external moments normal to the grip plane [13]. Finally, if it is required by the desired application, it can be also imposed that the current surface reconstruction center of mass (that is equivalent to the reconstructed object center of mass at the end of the process) has to be contained in the current grasping plane, enhancing the minimization of gravity and inertial effects during manipulation tasks [6], [16].

To do this, a field of forces defined as the sum of suitable force contributions is associated at each contact point.

First, the interpolating plane $\Pi$ of the current contact points -i.e., the plane which minimizes the distance from all the contact points-, and the projections $\boldsymbol{p}_{i}^{\Pi}$ of the contact point $\boldsymbol{p}_{i}$ on $\Pi$, with $i=1, \ldots, n_{f}$, are computed (see Fig. 5). Then, the force associated to the $i$-th contact point is:

$$
\boldsymbol{f}_{i}=\boldsymbol{f}_{\Pi i}+\boldsymbol{f}_{c_{m}}+\boldsymbol{f}_{e i}+\boldsymbol{f}_{a i}+\boldsymbol{f}_{b i},
$$

where each contribution of force, with reference to Fig. 5, is defined as follows:

- $\boldsymbol{f}_{\Pi i}=k_{\Pi}\left(\boldsymbol{p}_{i}^{\Pi}-\boldsymbol{p}_{i}\right)$ is the force which moves $\boldsymbol{p}_{i}$ to $\boldsymbol{p}_{i}^{\Pi}$, so that all contact points belong to the same grasp plane;

- $\boldsymbol{f}_{c_{m}}=k_{c_{m}}\left(\boldsymbol{c}_{m}-\boldsymbol{c}_{m}^{\Pi}\right)$ is the force, equal for all the contact points, which attracts the grasp plane $\Pi$ to $c_{m}$, where $\boldsymbol{c}_{m}$ is the center of mass of the current reconstruction surface and $\boldsymbol{c}_{m}^{\Pi}$ is the projection of the center of mass on the interpolating plane;

- $\boldsymbol{f}_{e i}=k_{e}\left(\theta_{i}-\frac{2 \pi}{n_{f}}\right) \boldsymbol{t}_{i}$ is the tangential force which is in charge of producing an equilateral grasp configuration, where $\theta_{i}$ is the angle between the vectors $\boldsymbol{p}_{i}^{\Pi}-\boldsymbol{c}_{m}^{\Pi}$ and $\boldsymbol{p}_{j}^{\Pi}-\boldsymbol{c}_{m}^{\Pi}$, with $j=i+1$ for $i=1, \ldots, n_{f}-1$, and $j=1$ for $i=n_{f}$, and $\boldsymbol{t}_{i}$ is the tangential unit vector

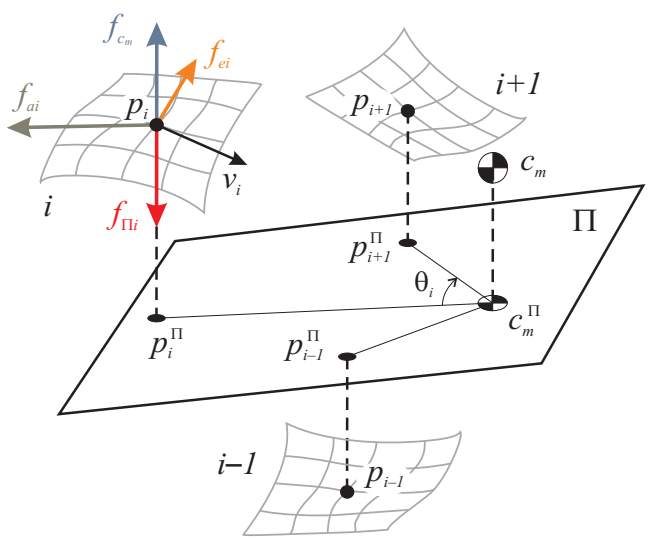

Fig. 5. Force field for the $i$-th floating contact point.

normal to $\boldsymbol{c}_{m}^{\Pi}-\boldsymbol{p}_{i}^{\Pi}$, lying on $\Pi$ and pointing toward $\boldsymbol{p}_{j}^{\Pi}$;

- $\boldsymbol{f}_{a i}=k_{a}\left(\boldsymbol{p}_{i}^{\Pi}-\boldsymbol{c}_{m}^{\Pi}\right) /\left\|\boldsymbol{p}_{i}^{\Pi}-\boldsymbol{c}_{m}^{\Pi}\right\|$ is a force component which tends to enlarge the area of the grasp polygon;

- $f_{b i}$ represents the kinematic barrier force, depending on the local and global kinematic index, described in the next subsection.

The parameters $k_{\Pi}, k_{c_{m}}, k_{e}, k_{a}$ are positive constant coefficients, suitably designed to weigh the single force contributions with respect to the requirements of the single situations and/or tasks to accomplish.

The force $\boldsymbol{f}_{i}$ is then projected onto the tangential plane to the current reconstruction surface at the contact point $i$, determining the direction of motion for the $i$-th contact point:

$$
\boldsymbol{f}_{i}^{\prime}=\boldsymbol{f}_{i}-\left(\boldsymbol{f}_{i}^{T} \boldsymbol{v}_{i}\right) \boldsymbol{v}_{i}
$$

where $\boldsymbol{v}_{i}$ is the unit normal vector to the reconstruction surface at the point $\boldsymbol{p}_{i}$.

The direction of $\boldsymbol{f}_{i}^{\prime}$ individuates one of the points of the surface close to the current one, as shown in Fig. 3, employed by the planner to produce the floating motion of the finger. When $\left\|\boldsymbol{f}_{i}^{\prime}\right\|$ is higher than a given threshold $\sigma_{f}$, the current grasp configuration changes according to the directions of $\boldsymbol{f}_{i}^{\prime}$. The choice of $\sigma_{f}$ means that forces $\boldsymbol{f}_{i}^{\prime}$ whose norm is under this threshold can be neglected, and when this happens for all the contact points, then the reached configuration is the local optimal grasp for the current iteration. Obviously $\sigma_{f}$ affects both the accuracy of the grasp solution and the computational time, determining the number of iterations required to converge to the local optimum, and thus it must be suitably tuned considering this trade-off.

\section{B. Kinematic barrier forces}

The kinematic barrier force $\boldsymbol{f}_{b i}$ for the $i$-th floating contact point is aimed at avoiding the motion of the finger along directions that cause the reaching of joint limits, joint or hand singularities, and collisions between fingers or with the palm. In detail, the barrier force is equal to

$$
\boldsymbol{f}_{b i}=\boldsymbol{f}_{j i}+\boldsymbol{f}_{s i}+\boldsymbol{f}_{c i},
$$


where each term is related to one of the neighbor points of the contour, directed from the corresponding contour point towards the actual contact point:

- $\boldsymbol{f}_{j i}$ is zero when the finger joint positions are far from their limits, while it quickly increases its magnitude, with a hyperbolic law, when one or more joint limits are approached at least for one of the contour points. Therefore, the force $f_{j i}$ is in charge to move the contact point far from unreachable positions.

- $f_{s i}$ is zero when the finger configuration is far from kinematic singularities, while it quickly increases, with a hyperbolic law, when a kinematic singularity is approached. Therefore, $\boldsymbol{f}_{s i}$ represents a force that is repulsive with respect to the directions leading to finger singularities.

- $f_{c i}$ is zero when the fingers are far from each others and from the palm, while its magnitude is increased when a safety distance is violated.

When the sum of each contribution in (5) for a finger results in a zero force field, the corresponding contact point does not change its position in the actual step of the current iteration of the planner stage. Notice that the barrier forces can be also employed to cope with environmental constraints, e.g. object ground plane or other surrounding objects.

\section{Finger trajectory planner}

The local grasp planner produces a sequence of intermediate target grasp configurations at each iteration of the object reconstruction algorithm, which ends with the optimal grasp configuration (in local sense). The intermediate configurations are used to generate the fingertip paths.

Namely, the sequence of intermediate configurations is suitably filtered by a spatial low-pass filter in order to achieve a smooth path for the fingers on the object surface. Notice that only the final configuration needs to be reached exactly, while the intermediate configurations can be considered as via points for finger trajectory generation, that can be computed in real-time with a one step delay.

With respect to the smooth paths through the points of the filtered configurations, the actual finger paths generated by the finger trajectory planner keep a distance $\delta_{f}$ along the normal to the surface. When the final configuration is reached, the safety distance is progressively reduced to zero, producing the desired grasp action, with directions of grasp perpendicular to the object reconstructed surface.

\section{EXPERIMENTS}

The proposed method has been experimentally tested on different objects considering a different number of fingers of the robotic hand of Fig. 4. The results for the objects shown in Fig. 2, a teddy-bear and a little bottle, are presented.

Images in a number equal to $n=13$ have been taken for both the objects by a common webcam mounted in inside the palm of the hand in Fig. 4. The reconstruction surface is sampled with $n_{s}=1500$ points, while the reconstruction parameters have been chosen as: $m=10^{-3} \mathrm{~kg}, k=0.3$. $10^{-3} \mathrm{~N} / \mathrm{m}, b=0.09 \cdot 10^{-3} \mathrm{Ns} / \mathrm{m}$, and $F_{a}=5 \mathrm{~N}$. For the
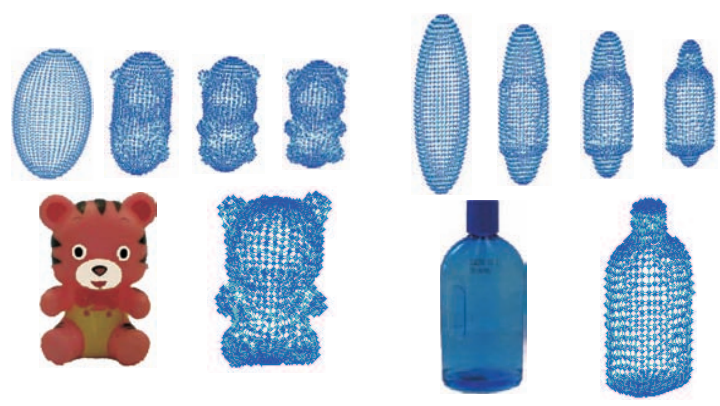

Fig. 6. Steps of the object surface reconstruction process.

grasp planner, $k_{\Pi}, k_{c_{m}}, k_{e}, k_{a}$ have been chosen all equal to 1 , so as to have an equivalent weight for all the contributions, while the threshold $\sigma_{f}$ has been tuned to a value of $0.002 \mathrm{~N}$. The floating security distance $\delta_{f}$ has been set to $2 \mathrm{~cm}$, which is deliberately a huge value for a better visualization of the trajectories. The computational time for the whole process is about $1.5 \mathrm{~s}$ on a Pentium $1.7 \mathrm{GHz}$.

In Fig. 6 some intermediate steps of the reconstruction algorithm are shown, while the finger trajectories and the final grasp configurations, respectively for the teddy-bear and for the little bottle, are shown in Fig. 7. Both cases of $k_{c_{m}}=$ 1 (left) and $k_{c_{m}}=0$ (right) are considered (the bold point represents the position of the object center of mass of the reconstructed object). In particular, for the case $k_{c_{m}}=1$ it is evident that both the grasp planes of the final grasps contain the center of mass of the objects, while for the case $k_{c_{m}}=0$ the plane of the final grasp is far from the center of mass to achieve a more extended areas of the grasp polygon.

More in detail, Figure 7 shows how the teddy-bear is grasped with three fingers achieving a desirable stable planar equilateral grasp ( $120^{\circ}$ apart) for both cases of $k_{c_{m}}=1$ and $k_{c_{m}}=0$. The yellow lines represent the sequence of reconstruction points selected by the planner during the evolution of the reconstruction surface. The green lines represent the trajectories that the planner generate for the fingertips after spatial filtering and considering the safety distance. Finally, the red lines show the last part of the grasp trajectory, when the safety distance is progressively reduced achieving a perpendicular approach to the object surface.

For the case of the little bottle, four fingers of the hand has been considered. The final grasp configuration corresponds to the equilateral best grasp $\left(90^{\circ}\right.$ apart) for the object. Moreover, the achieved trajectories are very regular due to the good choice of the initial grasp configuration evaluated by the preshaping module. This result is common when the object is symmetric with respect to one or more axes, and so it is well represented by an ellipsoidal surface. Of course, for the particular shape of the bottle, the results do not change significantly when $k_{c_{m}}$ is set to 0 .

Moreover, to validate the proposed method, a comparison between the results obtained with this approach and those obtained with traditional grasp quality indices has been performed. To make this comparison, the classical serial approach has been implemented. Hence, after the whole 


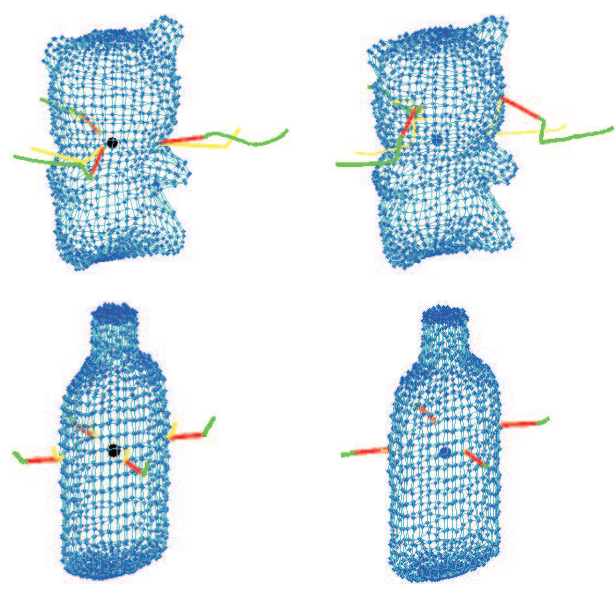

Fig. 7. Finger trajectories generated by the local grasp planner (green: approach, red: grasp) and the corresponding sequence of floating grasp points achieved during the reconstruction process (yellow) for two objects, both evaluated with $k_{c_{m}}=1$ (left) and $k_{c_{m}}=0$ (right).

TABLE I

COMPARISON USING TRADITIONAL GRASP QUALITY INDICES.

\begin{tabular}{|c|c|c|c|c|c|c|}
\cline { 2 - 7 } \multicolumn{1}{c|}{} & \multicolumn{2}{c|}{$Q_{1}$} & \multicolumn{2}{c|}{$Q_{2}$} & \multicolumn{2}{c|}{$Q_{3}$} \\
\cline { 2 - 7 } \multicolumn{1}{c|}{$k_{c_{m}}=1$} & Local & Global & Local & Global & Local & Global \\
\hline Bottle & 0.016 & 0.016 & 0.091 & 0.101 & 0.438 & 0.438 \\
\hline Teddy-Bear & 0.292 & 0.320 & 0.536 & 0.690 & 0.426 & 0.482 \\
\hline$k_{c_{m}}=0$ & Local & Global & Local & Global & Local & Global \\
\hline Bottle & 0.019 & 0.020 & 0.099 & 0.112 & 0.574 & 0.590 \\
\hline Teddy-Bear & 0.267 & 0.297 & 0.369 & 0.413 & 0.707 & 0.780 \\
\hline
\end{tabular}

reconstruction of the unknown object, a global search of the optimal grasp has been performed according to three quality indices: namely, $Q_{1}$ the max-min singular value of the grasp matrix [11], $Q_{2}$ the maximum volume of the ellipsoid in the wrench space [11] and $Q_{3}$ the largest perturbation wrench that the grasp can resist [9]. Where requested, a frictionless contact assumption has been done. Two further constraints have been included in the global search: grasp configurations which violated hand physical constraints and grasp configurations whose center of grip is far from the object center of mass have been both neglected. The latter has been obviously considered only in the case where $k_{c_{m}}=1$. Then, once the global optimal grasp has been found, the final grasp configuration obtained with the method proposed in this paper has been evaluated with each of the previous cited traditional grasp quality indices.

The results of this comparison are shown in Table I, where it is evident that the performances are very close in the case of the little bottle, while there is a small difference in the case of the teddy-bear. This result could be explained with the fact that the more regular is the object surface, the better are the results of the proposed local approach with respect to the global ones. Moreover, the evaluated final contact points in the global and in the local case are very close to each other, especially in the case of symmetric objects.

\section{CONCLUSION}

A method for fast visual grasping of unknown objects has been presented, which is composed of an iterative object surface reconstruction algorithm and of a local optimal grasp planner, evolving in a synchronized parallel way. The effectiveness of the proposed method has been confirmed by a number of case studies.

\section{REFERENCES}

[1] K. K. Aydin, "Fuzzy logic, grasp preshaping for robot hands", IEEE Annual Conference of the North America Fuzzy Information Processing Society, College Park,1995.

[2] C. Borst, M. Fischer and G. Hirzinger, "Calculating hand configurations for precision and pinch graps", IEEE/RSJ Conference on Intelligent Robots and Systems, Lausanne, 2002.

[3] M. Buss, H. Hashimoto and J. B. Moore, "Dexterous hand grasping force optimization", IEEE Transaction on Robotics and Automation, vol. 12, no.3, pp. 406-418, 1996

[4] E. Chinellato, R. B. Fisher, A. Morales and A. P. del Pobil, "Ranking planar grasp configurations for a three-fingered hand", IEEE International Conference on Robotics and Automation, Taipei, 2003.

[5] L. D. Cohen, "On active contour models and balloons", Computer Vision, Graphics, and Image Processing: Image Understanding, vol. 53, no. 2, pp. 211-229, 1991

[6] D. Ding, Y. H. Liu and S. Wang, "Computation of 3-D form-closure graps", IEEE Transaction on Robotics and Automation, vol. 17, no.4, pp. 512-522, 2001

[7] C. Dune, E. Marchand, C. Collowet and C. Leroux, "Active rough shape estimation of unknown objects", IEEE/RSJ International Conference on Intelligent Robots and Systems, Nice, 2008.

[8] C. R. Dyer, "Volumetric scene reconstruction from multiple views", Foundations of Image Analysis, L.S. Davis ed., Kluwer, Boston, 2001.

[9] C. Ferrari and J. Canny, "Planning optimal grasps", IEEE International Conference on Robotics and Automation, Nice, 1992.

[10] K. Huebner and D. Kragic, "Selection of robot pre-grasps using boxbased shape approximation", IEEE/RSJ International Conference on Intelligent Robots and Systems, Nice, 2008.

[11] Z. Li and S. S. Sastry, "Task-oriented optimal grasping by multifingered robot hands", IEEE Journal of Robotics and Automation, vol. 4, no. 1, pp. 32-44, 1988.

[12] V. Lippiello, F. Ruggiero and L. Villani, "Floating visual grasp of unknown objects", IEEE/RSJ International Conference on Intelligent Robots and Systems, St. Louis, 2009.

[13] B. Mirtich and J. Canny, "Easily computable optimum graps in 2-D and 3-D", IEEE International Conference on Robotics and Automation, San Diego, 1994.

[14] T. N. Nguyen and H. E. Stephanou, "A continuos model of robot hand preshaping", IEEE International Conference on Systems, Man and Cybernatics, Cambridge, 1989.

[15] D. Perrin, C. E. Smith, O. Masoud and N. P. Papanikolopoulos, "Unknown object grasping using statistical pressure models", IEEE International Conference on Robotics and Automation, San Francisco, 2000.

[16] J. Ponce and B. Faverjon "On computing three-finger force-closure grasps of polygonal objects", IEEE Transactions on Robotics and Automation, vol. 11, n.6, pp. 868-881, 1995

[17] M. Prats, P. J. Sanz and A. P. del Pobil "Task-oriented grasping using hand preshapes and tasks frames", IEEE International Conference on Robotics and Automation, Rome, 2007.

[18] K. B. Shimoga, "Robot grasp synthesis algorithms: A survey", International Journal of Robotics Research, vol. 15, n. 3, pp. 230266, 1996.

[19] R. Suarez, M. Roa and J. Cornella, "Grasp quality measures", Technical Report IOC-DT-P-2006-10, Universitat Politecnica de Catalunya, Institut d'Organitzacio i Control de Sistemes Industrials, 2006.

[20] T. Supuk, T. Kodek and T. Bajd, "Estimation of hand preshaping during human grasping", Medical Engineering \& Physics, vol. 27, pp. $790-797,2005$.

[21] S. A. Winges, D. J. Weber and M. Santello, "The role of vision on hand preshaping during reach to grasp", Experimental Brain Research, vol. 152, no. 4, pp. 489-498, 2003.

[22] B. H. Yoshimi and P. K. Allen, "Visual control of grasping and manipulation tasks", IEEE International Conference on Multisensor Fusion and Integration for Intelligent Systems, Las Vegas, 1994. 